\title{
A NOVEL STABILITY CONDITION AND ITS APPLICATION TO GA-BASED FUZZY CONTROL FOR NONLINEAR SYSTEMS WITH UNCERTAINTY
}

\author{
Po-Chen Chen \\ Department of Department of Civil Engineering, National Central University, Chung-li 320, Taiwan, R.O.C. \\ Cheng-Wu Chen \\ Department of Logistics Management, Shu-Te University, Kaohsiung 82445, Taiwan, R.O.C., cwchen@mail.stu.edu.tw \\ Wei-Ling Chiang \\ Department of Department of Civil Engineering, National Central University, Chung-li 320, Taiwan, R.O.C. \\ Ken Yeh \\ Department of Construction Science and Technology, De-Lin Institute of Technology, Taipei, Taiwan, R.O.C.
}

Follow this and additional works at: https://jmstt.ntou.edu.tw/journal

Part of the Civil and Environmental Engineering Commons

\section{Recommended Citation}

Chen, Po-Chen; Chen, Cheng-Wu; Chiang, Wei-Ling; and Yeh, Ken (2009) "A NOVEL STABILITY CONDITION AND ITS APPLICATION TO GA-BASED FUZZY CONTROL FOR NONLINEAR SYSTEMS WITH UNCERTAINTY," Journal of Marine Science and Technology. Vol. 17: Iss. 4, Article 6.

DOI: $10.51400 / 2709-6998.1985$

Available at: https://jmstt.ntou.edu.tw/journal/vol17/iss4/6

This Research Article is brought to you for free and open access by Journal of Marine Science and Technology. It has been accepted for inclusion in Journal of Marine Science and Technology by an authorized editor of Journal of Marine Science and Technology. 


\section{A NOVEL STABILITY CONDITION AND ITS APPLICATION TO GA-BASED FUZZY CONTROL FOR NONLINEAR SYSTEMS WITH UNCERTAINTY}

\section{Acknowledgements}

The authors would like to thank the National Science Council of the Republic of China, Taiwan, for their financial support of this research under Contract Nos. NSC 96-2628-E366-004-MY2 \& NSC

98-2221-E-366-006-MY2. The authors are also most grateful for the kind assistance of Prof. Chein-Shan Liu, Editor of JMST, and the constructive suggestions from anonymous reviewers all of which has led to the making of several corrections and suggestions that have greatly aided us in the presentation of this paper. 


\title{
A NOVEL STABILITY CONDITION AND ITS APPLICATION TO GA-BASED FUZZY CONTROL FOR NONLINEAR SYSTEMS WITH UNCERTAINTY
}

\author{
Po-Chen Chen*, Cheng-Wu Chen**, Wei-Ling Chiang* and Ken Yeh***
}

Key words: modified adaptive fuzzy sliding mode control, Lyapunov direct method, genetic algorithm.

\begin{abstract}
In this study, we strive to combine the advantages of fuzzy logic control (FLC), genetic algorithms (GA), $H_{\infty}$ tracking control schemes, smooth control and adaptive laws to design an adaptive fuzzy sliding model controller for the rapid and efficient stabilization of complex and nonlinear systems. First, we utilize a reference model and a fuzzy model (both involving FLC rules) to describe and well-approximate an uncertain, nonlinear plant. The FLC rules and the consequent parameter are decided on via GA. A boundary-layer function is introduced into these updated laws to cover modeling errors and to guarantee that the state errors converge into a specified error bound. After this, a $H_{\infty}$ tracking problem is characterized. We solve an eigenvalue problem (EVP), and derive a modified adaptive neural network controller (MANNC) to simultaneously stabilize and control the system and achieve $H_{\infty}$ control performance. Furthermore, a stability criterion is derived utilizing Lyapunov's direct method to ensure the stability of the nonlinear system. Finally, the control methodology is demonstrated via a numerical simulation.
\end{abstract}

\section{INTRODUCTION}

Over the past few years, there has been significant research efforts devoted to the analysis and control designs of fuzzy systems (see [2], [32], [34] and the references therein). The main motivation for this development has been applications to practical nonlinear systems and engineering problems (see [4, $10,13,15,17,24,25,27,29,30,31,37]$ and the references

Paper submitted 05/29/08; accepted 09/25/08. Author for correspondence: Cheng-Wu Chen (e-mail: cwchen@mail.stu.edu.tw).

*Department of Department of Civil Engineering, National Central University, Chung-li 320, Taiwan, R.O.C.

**Department of Logistics Management, Shu-Te University, Kaohsiung 82445, Taiwan, R.O.C.

***Department of Construction Science and Technology, De-Lin Institute of Technology, Taipei, Taiwan, R.O.C. therein). Undoubtedly, the Lyapunov theory is one of the most common approaches for dealing with the stability analysis of systems. However to overcome the conservatism that arises from the use of Lyapunov methods it has been necessary to develop a number of more effective methods, for example, fuzzy Lyapunov functions [8], [35]. There are also many important issues that have required studied for T-S fuzzy control systems, such as time delays $[4,7,9,26,42], \mathrm{H}_{\infty}$ performance [11, 20], robustness [23, 41], neural networks $(\mathrm{NN})$ and genetic algorithms (GA) [5, 14, 18, 28]. It should be noted that most of the aforementioned research efforts have been focused on PDC laws. Until now, how to integrate control theory and non-PDCs law into practical engineering computations has been an open question.

As has been explored in previous research, an adaptive algorithm can be adopted to find the best high-performance parameters for the fuzzy sliding mode controller (FSMC) [39], [40] for nonlinear control problems [19], [39]. Adaptive laws are devised afterwards to adjust the parameters of the fuzzy models, including the design of the adaptive fuzzy sliding mode controller (AFSMC) [43]. This is a powerful and robust control strategy for dealing with uncertainties and external disturbances.

Aside from the above, it is also very important to decide on the fuzzy rules and the initial values of the parameter vector values for the AFSMC. A GA [16], [22] is usually used as an optimization technique in the self-learning or training strategy for deciding on the fuzzy control rules and the initial values of the parameter vector. Using this GA-based AFSMC improves the immediate response, stability, and robustness of the control system. A new Lyapunov stability criterion needs to be developed for the $H_{\infty}$ tracking performance throughout all the system states to be considered in the proposed control strategy [3]. Based on the Lyapunov theory [33], it can be be shown that the proposed controller guarantees good $H_{\infty}$ tracking performance throughout the entire system states.

Another common problem encountered when switching the control input of the sliding model system is the so-called "chattering" phenomenon [38]. The smoothing of control discontinuity inside a thin boundary layer essentially acts as a low-pass filter structure for the local dynamics. This eliminates chattering, to guarantee that the state errors converge within a specified error bound. 
This study focuses on designing a robust tracking control for a class of nonlinear system involving plant uncertainties and external disturbances. To achieve this task, $H_{\infty}$ tracking control is incorporated into the AFSMC, to produce the proposed $H_{\infty}$ AFSMC design. It is shown that the proposed controller can guarantee $H_{\infty}$ tracking performance throughout the entire system states, based on the Lyapunov theory [33]. The Lyapunov stability condition is transformed into the form of a certain linear matrix inequality (LMI) problem $[1,21,36]$ which allows one to efficiently obtain the parameters of the controller by using the convex optimization techniques to solve either the EVP or the LMI problem.

\section{REFERENCE MODELING FOR NONLINEAR DYNAMIC SYSTEMS}

The plant is a single-input/single-out $n$ th-order system with $n \geq 1$.

$$
\stackrel{(n)}{y}=f(x)+g(x) \cdot u+d(t)
$$

The system is said to have a relative degree $n$, if $g(x)$ is bounded away from zero.

Assumption 1: $g(x)$ is bounded away from zero over a compact set $\zeta \subset R^{n},|g(x)| \geq b>0, \forall x \in \zeta$.

$$
r=\stackrel{(n)}{y}_{r}+\alpha_{n-1} \stackrel{(n-1)}{y_{r}}+\alpha_{n-2} \quad \stackrel{(n-2)}{y_{r}}+\ldots+\alpha_{1} \dot{y}_{r}+\alpha_{0} y_{r}
$$

where $\alpha_{n-1}, \ldots, \alpha_{1}, \alpha_{0}$ are chosen such that the polynomial $\ell^{n}+$ $\alpha_{n-1} \ell^{n-1}+\ldots+\alpha_{1} \ell+\alpha_{0}$ is Hurwitz; and $\ell$ here denotes the Laplace variable. If $f(x), g(x)$ are known, and Assumption 1 is satisfied, the control law can defined by

$$
u=\frac{-f(x)-d(x)-\left(\alpha_{n-1}{ }^{(n-1)} y+\ldots+\alpha_{1} \dot{y}+\alpha_{0} y\right)+r}{g(x)},
$$

$$
\forall x \in S
$$

Substituting (3) into (1), the linearized system becomes

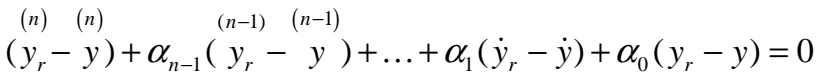

If we define $e=y_{r}-y$ as the tracking error, then the reference control input (2) results in the following error equation:

$$
\stackrel{(n)}{e}+\alpha_{n-1} \stackrel{(n-1)}{e}+\ldots+\alpha_{1} \dot{e}+\alpha_{0} e=0
$$

\section{GA-BASED $H_{\infty}$ MAFSMC FOR NONLINEAR SYSTEMS}

The control objective can be achieved by the control law

$$
u=u_{e q}+u_{d}
$$

where $u_{e q}$ is equivalent control. Now let

$$
u_{d}=-g(x)^{-1} \frac{1}{\rho}|S| \cdot \operatorname{sat}(S / \Phi)
$$

The thin boundary layer function $\operatorname{sat}(S / \Phi)$ is defined as

$$
\operatorname{sat}(S / \Phi)=\left\{\begin{array}{lll}
1, & \text { if } & (S / \Phi)>1 \\
(S / \Phi), & \text { if } & -1 \leq(S / \Phi) \leq 1 \\
-1, & \text { if } & (S / \Phi)<-1
\end{array}\right.
$$

We can utilize the certainty equivalent control approach [40] to design an FLC (8) to approximate $u_{e q}$

$$
\bar{u}_{e q}(\bar{\theta})=\sum_{k=1}^{m} R_{k}\left(-\left(\frac{\left\|S-C_{k}\right\|}{\beta}\right)^{2}\right) \cdot \bar{\theta}_{k}
$$

where $m$ is the sum of the fuzzy rules; $\bar{\theta}_{k}$, i.e, $\left|\bar{\theta}_{k}\right| \leq \theta_{\max }$ are adjustable consequent parameters of FLC; and $R(S)=\left[R_{1}(S)\right.$, $\left.R_{2}(S), \ldots, R_{m}(S)\right]^{T}$ is the vector of the fuzzy basis function [31] defined as

$$
\begin{gathered}
R_{k}=\frac{\mu_{k}\left(S-C_{k}\right)}{\sum_{k=1}^{m} \mu_{k}\left(S-C_{k}\right)}, \\
\mu_{k}\left(\left\|S-C_{k}\right\|\right)=\exp \left(-\left(\frac{\left\|S-C_{k}\right\|}{\beta}\right)^{2}\right)
\end{gathered}
$$

\section{Assumption 2:}

For $x \in \zeta \subset R^{n}$, there exists an adjustable parameter vector $\bar{\theta}=\left[\bar{\theta}_{1}, \bar{\theta}_{2}, \cdots, \bar{\theta}_{n}\right]^{T}$ such that the fuzzy system $\bar{u}(S, \bar{\theta})=$ $\bar{\theta}^{T} R(S)$ can approximate the continuous function $u$ with accuracy $\varepsilon_{\max }$ over the set $\zeta$, that is, $\exists \bar{\theta}$ such that

$$
\sup \left|\bar{u}_{e q}(S, \bar{\theta})-u_{e q}(S)\right| \leq \varepsilon_{\max }, \forall S \in \zeta
$$

Let $\hat{\theta}$ denote the estimative of $\bar{\theta}$ at time t. Then, define the estimative control output $\hat{u}_{e q}(S, \hat{\theta})$ as 


$$
\hat{u}_{e q}(S, \hat{\theta})=\sum_{k=1}^{m} \hat{\theta}_{k} \cdot R_{k}(S)=\hat{\theta}^{T} R(S)
$$

If the parameter error vector at time $\mathrm{t}$ is $\tilde{\theta}=\bar{\theta}-\hat{\theta}$, then,

$$
\tilde{\theta}^{T} R(S)=\bar{u}_{e q}(S, \bar{\theta})-\hat{u}_{e q}(S, \hat{\theta})
$$

From Assumption 2, define the modeling error as follows:

$$
\varepsilon=u_{e q}-\bar{u}_{e q}(S, \bar{\theta}) \text {, where }|\varepsilon| \leq \varepsilon_{\max }
$$

We can now say

$$
u_{e q}=\hat{u}_{e q}(S, \hat{\theta})+\tilde{\theta}^{T} R(S)+\varepsilon
$$

Then, substituting (7) and (14) into (3), we can obtain the error dynamic equation from

$$
\begin{aligned}
\stackrel{(n)}{e}+\alpha_{n-1} & \stackrel{(n-1)}{e}+\ldots+\alpha_{1} \dot{e}+\alpha_{0} e \\
= & g(x) \cdot\left(\tilde{\theta}^{T} R(S)+\varepsilon\right)-\frac{1}{\rho}|S| \cdot \operatorname{sat}(S / \Phi)
\end{aligned}
$$

Define the augmented error as

$$
S=\beta_{n-1} \stackrel{(n-1)}{e}+\ldots+\beta_{1} \dot{e}+\beta_{0} e
$$

where $\beta_{n-1}, \ldots, \beta_{1}, \beta_{0}$ in (15); and $\alpha_{n-1}, \ldots, \alpha_{1}, \alpha_{0}$ in (14) are chosen such that

$$
\hat{M}(\ell)=\frac{\beta_{n-1} \ell^{n-1}+\ldots+\beta_{1} \ell+\beta_{0}}{\ell^{n}+\alpha_{n-1} \ell^{n-1}+\ldots+\alpha_{1} \ell+\alpha_{0}}=\frac{N(\ell)}{D(\ell)}
$$

is SPR (strictly positive real) transfer function, and $N(\ell)$ and $D(\ell)$ are coprime.

Define $e_{m}=[e, \dot{e}, \cdots, \stackrel{(n-1)}{e}]^{T}$ as the states for (16) which can be realized as

$$
\dot{e}_{m}(t)=\Lambda \cdot e_{m}(t)+b \cdot\left[g(x) \cdot\left(\tilde{\theta}^{T} R(S)+\varepsilon\right)-\frac{1}{\rho}|S| \cdot \operatorname{sat}(S / \Phi)\right]
$$

$$
S(t)=c^{T} e_{m}(t)
$$

Where

$$
\Lambda=\left[\begin{array}{cccccc}
0 & 1 & 0 & \cdots & 0 & 0 \\
0 & 0 & 1 & \cdots & 0 & 0 \\
\vdots & \vdots & \vdots & \ddots & \vdots & \vdots \\
0 & 0 & 0 & \cdots & 1 & 0 \\
0 & 0 & 0 & \cdots & 0 & 1 \\
-\alpha_{0} & -\alpha_{1} & -\alpha_{2} & \cdots & -\alpha_{n-2} & -\alpha_{n-1}
\end{array}\right]_{n \times n} \quad, b=\left[\begin{array}{c}
0 \\
0 \\
\vdots \\
0 \\
0 \\
1
\end{array}\right]_{n \times 1}
$$

$c=\left[\begin{array}{llll}\beta_{0} & \beta_{1} & \cdots & \beta_{n-1}\end{array}\right]^{T}$, let $\beta_{n-1}=1$.

Observing (19), it is clear that the lumped uncertainty $\omega=$ $g(x) \cdot \mathcal{E}$ directly affects the tracking error. To achieve the control objective, the following $H_{\infty}$ tracking performance related to the tracking error vector $e_{m}$ is requested [36]:

$$
\int_{0}^{T} e_{m}^{T} Q e_{m} d t \leq e_{m}^{T}(0) P e_{m}(0)+\frac{1}{\gamma} \tilde{\theta}^{T}(0) H_{11} \tilde{\theta}(0)+\rho^{2} \int_{0}^{T} \omega^{2} d t
$$

for all $\omega \in L_{2}[0, T], \forall T \in[0, \infty)$, where $Q$ and $P$ are symmetric positive definite weighting matrices; and $0<\rho<1$ is a prescribed attenuation level.

It is seen that if the system starts with the initial conditions $e_{m}(0)=0, \tilde{\theta}(0)=0$, then the $H_{\infty}$ tracking performance of (21) becomes

$$
\int_{0}^{T} e_{m}^{T} Q e_{m} d t \leq \rho^{2} \int_{0}^{T} \omega^{2} d t
$$

That is, the $L_{2}$-gain from $\omega$ to $e_{m}$ must be equal to or less than a prescribed value $\rho$ [36]. The following results can be obtained:

Theorem 1: Consider the nonlinear uncertain system $\stackrel{(n)}{y}=$ $f(x)+g(x) \cdot u+d$ that satisfies Assumptions $(\bar{\theta}, \hat{\theta})$. Suppose that the unknown control input $u$ which is approximated by $\hat{u}_{e q}(S, \hat{\theta})$ by (11), $S$ is given by (17), $Q$ is a symmetric positive definite weighting matrix, and $0<\rho<1$ is the design constant serving as an attenuation level.

Let $K=K^{T}>0$ be the solution of the following LMI:

$$
\Lambda^{T} K+K \Lambda+\frac{1}{\rho} K b b^{T} K-\frac{1}{\rho} c c^{T}+\frac{1}{\rho} Q \leq 0
$$

and define $P=c c^{T}+K$. The control law chosen is $u_{e q}(S, \hat{\theta})$ and the modified adaptive laws are given by

$$
\dot{\hat{\theta}}=\gamma \cdot|S| \cdot \operatorname{sat}(S / \Phi) R+\gamma \cdot b^{T} K e_{m} R
$$


in which $\gamma>0$ is the adaptive gain. The $H_{\infty}$ tracking performance of (21) for the overall system can be guaranteed.

\section{Proof:}

The time derivative of $S$ can be obtained by

$$
\begin{aligned}
\dot{S}=c^{T} \dot{e}_{m}(t)= & c^{T}\left\{\Lambda \cdot e_{m}(t)+b \cdot\left[g(x) \cdot\left(\tilde{\theta}^{T} R(S)+\varepsilon\right)\right.\right. \\
& \left.\left.-\frac{1}{\rho}|S| \cdot \operatorname{sat}(S / \Phi)\right]\right\}
\end{aligned}
$$

Now, we shall investigate the stability of the origin using Lyapunov function candidates. The Lyapunov function candidate of the state is defined as follows:

$$
V=\frac{1}{2} e_{m}^{T} P e_{m}+\frac{1}{2 \gamma} \tilde{\theta}^{T} H \tilde{\theta}
$$

where $\gamma$ is a positive constant representing the learning rate,

$$
\tilde{\theta}=\left[\begin{array}{llll}
\tilde{\theta}_{1} & \tilde{\theta}_{2} & \cdots & \tilde{\theta}_{m}
\end{array}\right]^{T}, H=g(x) \cdot \mathrm{I}_{m \times m},
$$

because $P=c c^{T}+K,(26)$ can be expressed as

$$
\begin{aligned}
V & =\frac{1}{2} e_{m}^{T}\left(c c^{T}+K\right) e_{m}+\frac{1}{2 \gamma} \tilde{\theta}^{T} H \tilde{\theta} \\
& =\frac{1}{2}\left(S^{2}+e_{m}^{T} K e_{m}\right)+\frac{1}{2 \gamma} \tilde{\theta}^{T} H \tilde{\theta}
\end{aligned}
$$

The time derivative of $V$ can be described as

$$
\begin{aligned}
\dot{V}= & S \dot{S}+\frac{1}{2} \dot{e}_{m}^{T} K e_{m}+\frac{1}{2} e_{m}^{T} K \dot{e}_{m}+\frac{1}{\gamma} \tilde{\theta}^{T} H \dot{\tilde{\theta}} \\
= & S \cdot\left[g(x) \cdot \tilde{\theta}^{T} R(S)\right]+S \cdot[g(x) \cdot \varepsilon]-\frac{1}{\rho} S^{2} \\
& +\frac{1}{2} e_{m}^{T}\left(\Lambda^{T} K+K \Lambda\right) e_{m}+\left[g(x) \cdot\left(\tilde{\theta}^{T} R(S)+\varepsilon\right)\right. \\
& \left.-\frac{1}{\rho}|S| \cdot \operatorname{sat}(S / \Phi)\right] \cdot b^{T} K e_{m}+\frac{1}{\gamma} \tilde{\theta}^{T} H(-\dot{\hat{\theta}})
\end{aligned}
$$

Substituting the adaptive laws (24) into (27), and when $|S|>\Phi$, we derive

$$
\begin{aligned}
\dot{V}= & S \cdot \omega-\frac{1}{\rho} S^{2}+\frac{1}{2} e_{m}^{T}\left(\Lambda^{T} K+K \Lambda\right) e_{m}+\omega \cdot b^{T} K e_{m} \\
& -\frac{1}{\rho} S \cdot b^{T} K e_{m}
\end{aligned}
$$

(27) can now be rewritten as

$$
\begin{aligned}
\dot{V}= & \omega \cdot\left(S+b^{T} K e_{m}\right)-\frac{1}{\rho} S^{2}+\frac{1}{2} e_{m}^{T}\left(\Lambda^{T} K+K \Lambda\right) e_{m}-\frac{1}{\rho} S \cdot b^{T} K e_{m} \\
= & -\left[\frac{1}{\sqrt{2}} \sqrt{\rho} \cdot \omega-\frac{1}{\sqrt{2 \rho}}\left(S+b^{T} K e_{m}\right)\right]^{2}+\frac{1}{2} \rho \omega^{2} \\
& +\frac{1}{2 \rho}\left(S+b^{T} K e_{m}\right)^{2}-\frac{1}{\rho} S^{2}+\frac{1}{2} e_{m}^{T}\left(\Lambda^{T} K+K \Lambda\right) e_{m} \\
& -\frac{1}{\rho} S \cdot b^{T} K e_{m} \\
= & -\left[\frac{1}{\sqrt{2}} \sqrt{\rho} \cdot \omega-\frac{1}{\sqrt{2 \rho}}\left(S+b^{T} K e_{m}\right)\right]^{2}+\frac{1}{2} \rho \omega^{2} \\
& +\frac{1}{2 \rho}\left(S^{2}+2 S b^{T} K e_{m}+e_{m}^{T} K b b^{T} K e_{m}\right) \\
& -\frac{1}{\rho} S^{2}+\frac{1}{2} e_{m}^{T}\left(\Lambda^{T} K+K \Lambda\right) e_{m}-\frac{1}{\rho} S \cdot b^{T} K e_{m}
\end{aligned}
$$

$$
\begin{aligned}
& \leq \frac{1}{2} e_{m}^{T}\left(\Lambda^{T} K+K \Lambda+\frac{1}{\rho} K b b^{T} K\right) e_{m}-\frac{1}{2 \rho} S^{2}+\frac{1}{2} \rho \omega^{2} \\
& =\frac{1}{2} e_{m}^{T}\left(\Lambda^{T} K+K \Lambda+\frac{1}{\rho} K b b^{T} K-\frac{1}{\rho} c c^{T}\right) e_{m}+\frac{1}{2} \rho \omega^{2}
\end{aligned}
$$

From (22),

$$
\dot{V} \leq-\frac{1}{2 \rho} e_{m}^{T} Q e_{m}+\frac{1}{2} \rho \omega^{2}
$$

Integrating both sides of (28) yields

$$
V\left(t_{f}\right)-V(0) \leq-\frac{1}{2 \rho} \int_{0}^{t_{f}} e_{m}^{T} Q e_{m} d t+\frac{\rho}{2} \int_{0}^{t_{f}} \omega^{2} d t
$$

Thus,

$$
\int_{0}^{t_{f}} e_{m}^{T} Q e_{m} d t \leq 2 \rho V(0)-2 \rho V\left(t_{f}\right)+\rho^{2} \int_{0}^{t_{f}} \omega^{2} d t
$$

Since $0<\rho<1$ and $V \geq 0,(31)$ can be rewritten as

$$
\int_{0}^{t_{f}} e_{m}^{T} Q e_{m} d t \leq 2 V(0)+\rho^{2} \int_{0}^{t_{f}} \omega^{2} d t
$$

With (25), the following result can be obtained:

$$
\int_{0}^{t_{f}} e_{m}^{T} Q e_{m} d t \leq e_{m}^{T}(0) P e_{m}(0)+\frac{1}{\gamma} \tilde{\theta}^{T}(0) H_{11} \tilde{\theta}(0)+\rho^{2} \int_{0}^{t_{f}} \omega^{2} d t
$$

So, the $H_{\infty}$ tracking performance in (18) is achieved. This completes the proof. 
Note that the matrix inequalities in (22) can be transformed into a certain form of LMI. That is, by the Schur complements [1], (22) is equivalent to

$$
\left[\begin{array}{ccc}
\Lambda^{T} K+K \Lambda-\frac{1}{\rho} c c^{T} & K b & Q^{1 / 2} \\
b^{T} K & -\rho & 0_{1 \times n} \\
\left(Q^{1 / 2}\right)^{T} & 0_{n \times 1} & -\rho \cdot \mathrm{I}_{n \times n}
\end{array}\right] \leq 0
$$

where $\left(Q^{1 / 2}\right)^{T} Q^{1 / 2}=Q$. Therefore, the minimization problem can be formulated as the following eigenvalue problem (EVP): subject to $K_{i}=K_{i}^{T}>0$ and (32).

\section{NUMERICAL SIMULATION}

The active TMD mounted on a shear structure is modeled as a Four-degree-of freedom structure-ATMD system, as shown in Fig. 1 ATMD System. The parameters with ATMD: $m_{d}=50(\mathrm{~kg}), c_{d}=14.05(\mathrm{~N}-\mathrm{s} / \mathrm{m})$, and $k_{d}=9875.18(\mathrm{~N} / \mathrm{m})$ represent mass, damping, and stiffness, and the parameter vectors: $M_{S T}(\mathrm{~kg}), C_{S T}(t)(\mathrm{N}-\mathrm{s} / \mathrm{m})$, and $K_{S T}(t)(\mathrm{N} / \mathrm{m})$ represent the mass, uncertain damping, and uncertain stiffness vectors, respectively, $(t=t+\Delta t, \Delta t=0.02 \mathrm{sec})$ of this dilapidatedsimulation structure with ATMD shown below. $F$ and $u$ represent the external force and control input. The dynamic equations of motion of the pendulum are given below.

$$
\begin{aligned}
& {\left[\begin{array}{l}
\ddot{x}_{1} \\
\ddot{x}_{2} \\
\ddot{x}_{3} \\
\ddot{x}_{d}
\end{array}\right]=\left[\begin{array}{ll}
\left(-M^{-1} K_{S T}\right)_{4 \times 4} & \left(-M^{-1} C_{S T}\right)_{4 \times 4}
\end{array}\right]\left[\begin{array}{c}
x_{1} \\
x_{2} \\
x_{3} \\
x_{d} \\
\dot{x}_{1} \\
\dot{x}_{2} \\
\dot{x}_{3} \\
\dot{x}_{d}
\end{array}\right]+\left[\begin{array}{c}
0 \\
0 \\
-1 / m_{3} \\
-1 / m_{d}
\end{array}\right] \cdot u} \\
& +\left[\begin{array}{c}
-1 / m_{1} \\
-1 / m_{2} \\
-1 / m_{3} \\
-1 / m_{d}
\end{array}\right] \cdot F
\end{aligned}
$$

where $M_{S T}=\left[\begin{array}{cccc}980.71 & 0 & 0 & 0 \\ 0 & 980.71 & 0 & 0 \\ 0 & 0 & 980.71 & 0 \\ 0 & 0 & 0 & 50\end{array}\right]$,

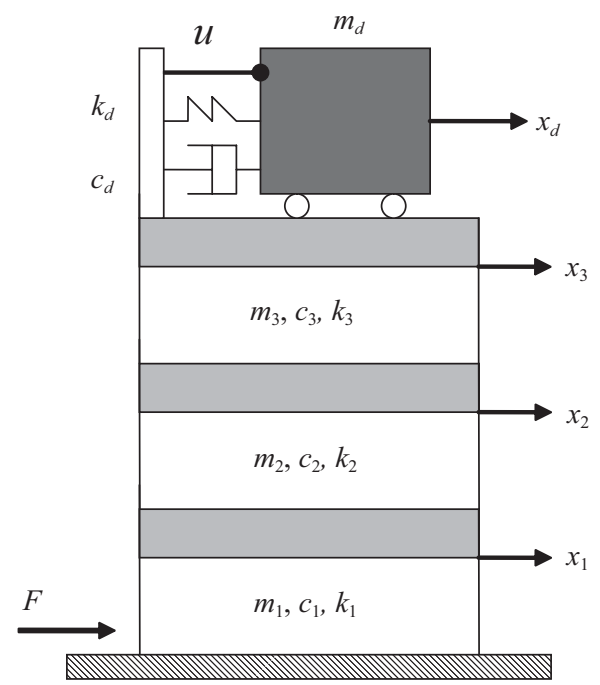

Fig. 1. Four-DOF structure-ATMD system.

$$
\begin{aligned}
K_{S T}(t) & =\left[\begin{array}{cccc}
2740599(1-0.3 t) & -1640938(1-0.3 t) & 368992(1-0.3 t) & 0 \\
-1640938(1-0.3 t) & 3020937(1-0.3 t) & -1624126(1-0.3 t) & 0 \\
368992(1-0.3 t) & -1624126(1-0.3 t) & 1333065(1-0.3 t)+9875.2 & -9875.2 \\
0 & 0 & -9875.2 & 9875.2
\end{array}\right] \\
C_{S T}(t) & =\left[\begin{array}{cccc}
382.65(1+0.1 t) & -57.27(1+0.1 t) & 61.64(1+0.1 t) & 0 \\
-57.27(1+0.1 t) & 456.73(1+0.1 t) & -2.63(1+0.1 t) & 0 \\
61.64(1+0.1 t) & -2.63(1+0.1 t) & 437.29(1+0.1 t)+14.05 & -14.05 \\
0 & 0 & -14.05 & 14.05
\end{array}\right]
\end{aligned}
$$

The design procedure for the GA-based $H_{\infty}$-MAFSMC analyzed above can be summarized as follows:

[Step 1] In the simulation we set the sensor on the top floor of a dilapidated structure. Using the procedure discussed above, we specify the response of the control system by defining suitable sliding surface $S=c^{T} e_{m}=30 e_{3}+\dot{e}_{3}$, then, construct the structure of the MAFSMC based on GA and the initial values of the consequent parameter vector $\hat{\theta}$ chosen as follows: [ $\begin{array}{llllllllll}1 & 0.9 & 0.65 & 0.30 & 0.05 & 0 & -0.06 & -0.25 & -0.33 & -0.7\end{array}$ $-0.95]$ '.

[Step 2] Let $Q=\mathrm{I}_{2 \times 2}$, with the specified attenuation level $\rho=$ 0.5. Now we use the LMI optimization toolbox in Matlab [21] to solve the EVP in (32), to obtain $K=\left[\begin{array}{cc}232.4346 & -13.2239 \\ -13.2239 & 2.7998\end{array}\right]$ and $P=c c^{T}+K=\left[\begin{array}{cc}1132.4346 & 16.7761 \\ 16.7761 & 3.7998\end{array}\right]$.

[Step 3] Apply the controller, as given in (7) and (14), to control the nonlinear system (1). Next, we let $\gamma=0.05, \Phi=0.2$, and adjust $\hat{\theta}$ by the adaptive law, as given by (23). 


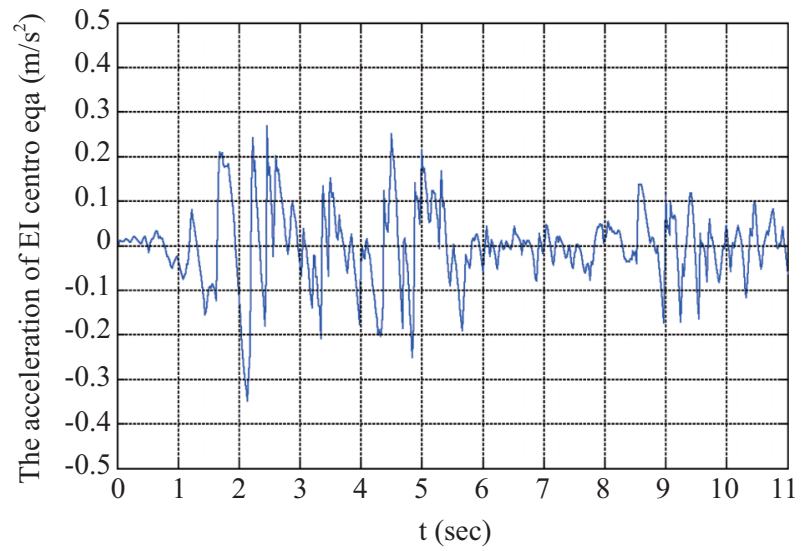

Fig. 2. Acceleration of the El Centro earthquake.

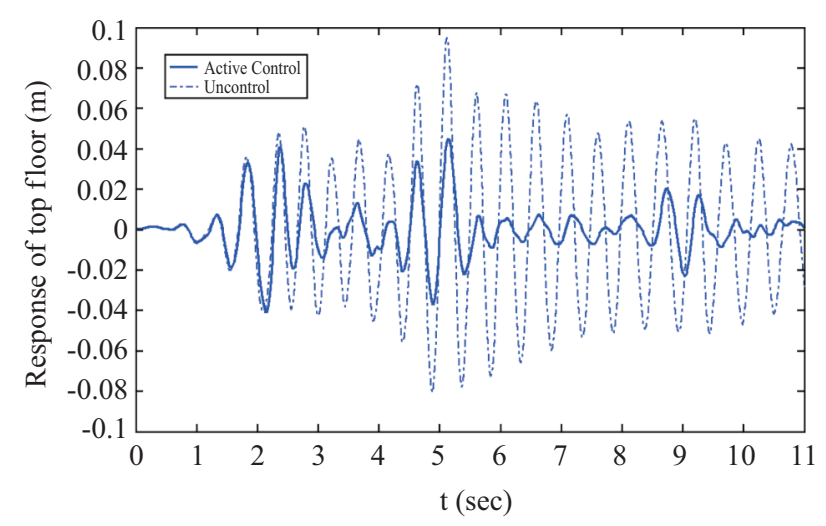

Fig. 3. Top floor response of the structure in the El Centro earthquake.

An examination of the simulation results for the El Centro earthquake disturbances, illustrated in Figs. 2-3, shows an improvement in the performance of the system in all respects while retaining the advantage of robustness in the presence of bounded disturbances.

Figures 2-3 show that the dilapidated structure simulation is rapidly stable and the GA-based $H_{\infty}$-MAFSMC can be derived to stabilize and control the system.

\section{CONCLUSIONS}

A GA-based $H_{\infty}$ modified adaptive fuzzy sliding model controller for the handling the nonlinear systems is described in this paper. We first track the reference trajectory to approximate an uncertain and nonlinear plant via fuzzy model involving FLC rules. Then, we utilize a GA to decide on the initial values of the consequent parameter vector $\hat{\theta}$. The $H_{\infty}$ tracking problem is characterized in terms of solving an eigenvalue problem. The smoothing of control discontinuities inside a thin boundary layer, essentially as a low-pass filter structure, is introduced into these updated laws for the elimination of chattering and to guarantee that the state errors converge within a specified error bound. Next, an MAFSMC is proposed to stabilize the system, and achieve $H_{\infty}$ control performance at the same time. A stability criterion is also derived from Lyapunov's direct method to ensure the stability of the nonlinear system. The stability analysis can thus be reduced into LMI problems. Finally, we discuss an example, a numerical simulation. We demonstrate that the control methodology can rapidly and efficiently control an uncertain and nonlinear system.

\section{ACKNOWLEDGMENT}

The authors would like to thank the National Science Council of the Republic of China, Taiwan, for their financial support of this research under Contract Nos. NSC 96-2628-E366-004-MY2 \& NSC 98-2221-E-366-006-MY2. The authors are also most grateful for the kind assistance of Prof. Chein-Shan Liu, Editor of JMST, and the constructive suggestions from anonymous reviewers all of which has led to the making of several corrections and suggestions that have greatly aided us in the presentation of this paper.

\section{REFERENCES}

1. Boyd, S., Ghaoui, E., Feron, E., and Balakrishnan, V., Linear Matrix Inequalities in System and Control Theory, Philadelphia, PA, SIAM (1994).

2. Chang, W. J., Yeh, Y. L., and Meng, Y. T., "State variance constrained fuzzy constrained fuzzy control via observer-based fuzzy controllers," Journal of Marine Science and Technology, Vol. 14, No. 1, pp. 49-57 (2006).

3. Chen, B. S., Lee, C. H., and Chang, Y. C., " $H_{\infty}$ tracking design of uncertain nonlinear SISO systems: adaptive fuzzy approach," IEEE Transactions on Fuzzy Systems, Vol. 4, No. 1, pp. 32-43 (1996).

4. Chen, B., Liu, X. P., and Tong, S. C., "New delay-dependent stabilization conditions of T-S fuzzy systems with constant delay," Fuzzy Sets and Systems, Vol. 158, No. 20, pp. 2209-2224 (2007).

5. Chen, C. W., "Modeling and control for nonlinear structural systems via a NN-based approach,” Expert Systems with Applications, Vol. 36, No. 3, pp. 4765-4772 (2009).

6. Chen, C. W., "The stability of an oceanic structure with T-S fuzzy models," Mathematics and Computers in Simulation, Vol. 80, No. 2, pp. 402 426 (2009).

7. Chen, C. W., Chiang, W. L., and Hsiao, F. H., "Stability analysis of T-S fuzzy models for nonlinear multiple time-delay interconnected systems," Mathematics and Computers in Simulation, Vol. 66, No. 6, pp. 523-537 (2004).

8. Chen, C. W., Chiang, W. L., Tsai, C. H., Chen, C. Y., and Wang, Morris H. L., "Fuzzy Lyapunov method for stability conditions of nonlinear systems," International Journal on Artificial Intelligence Tools, Vol. 15, No. 2, pp. 163-171 (2006).

9. Chen, C. W., Lin, C. L., Tsai, C. H., Chen, C. Y., and Yeh, K., "A novel delay-dependent criteria for time-delay T-S fuzzy systems using fuzzy Lyapunov method," International Journal on Artificial Intelligence Tools, Vol. 16, No. 3, pp. 545-552 (2007).

10. Chen, C. W., Shen, C. W., Chen, C. Y., and Cheng, M. J., "Stability analysis of an oceanic structure using the Lyapunov method," Engineering Computations, Vol. 27, (2010). (In press)

11. Chen, C. W., Yeh, K., Chiang, W. L., Chen, C. Y., and Wu, D. J., "Modeling, $\mathrm{H}^{\infty}$ control and stability analysis for structural systems using Takagi-Sugeno fuzzy model," Journal of Vibration and Control, Vol. 13, No. 11, pp. 1519-1534 (2007). 
12. Chen, C. W., Yeh, K., and Liu, F. R., “Adaptive fuzzy sliding mode control for seismically excited bridges with lead rubber bearing isolation," International Journal of Uncertainty, Fuzziness and KnowledgeBased Systems, Vol. 17, No. 5, pp. $705-727$ (2009).

13. Chen, C. W., Wang, Morris H. L., and Lin, J. W., "Managing target the cash balance in construction firms using a fuzzy regression approach," International Journal of Uncertainty, Fuzziness and Knowledge-Based Systems, Vol. 17, No. 5, pp. 667-684 (2009).

14. Chen, C. Y., Hsu, R. C., and Chen, C. W., "Fuzzy logic derivation of neural network models with time delays in subsystems," International Journal on Artificial Intelligence Tools, Vol. 14, No. 6, pp. 967-974 (2005).

15. Chen, C. Y., Shen, C. W., Chen, C. W., Liu, K. F. R., and Cheng, M. J., "A stability criterion for time-delay tension leg platform systems subjected to external force," China Ocean Engineering, Vol. 23, No. 1, pp. 49-57 (2009).

16. Chen, P. C., Genetic Algorithm for Control of Structure System, Masters Thesis, Department of Civil Engineering, Chung Yuan University, Taiwan (1998).

17. Chen, P. C., Chen, C. W., and Chiang, W. L., "GA-based fuzzy sliding mode controller for nonlinear systems," Mathematical Problems in Engineering, Vol. 2008, Doi: 10.1155/2008/325859 (2008).

18. Chen, P. C., Chen, C. W., and Chiang, W. L., "GA-based modified adaptive fuzzy sliding mode controller for nonlinear systems," Expert Systems with Applications, Vol. 36, No. 3, pp. $5872-5879$ (2009).

19. Feng, G., Cao, S. G., and Rees, N. W., "Stable adaptive control of fuzzy dynamic systems," Fuzzy Sets and Systems, Vol. 131, No. 2, pp. 217-224 (2002).

20. Feng, G., Chen, C.-L., Sun, D., and Zhu, Y., " $\mathrm{H}^{\infty}$ controller synthesis of fuzzy dynamic systems based on piecewise Lyapunov functions and bilinear matrix inequalities," IEEE Transactions on Fuzzy Systems, Vol. 13, No. 1, pp. 94-103 (2005).

21. Gahinet, P., Nemirovski, A., Laub, A., and Chilali, M., LMI Control Toolbox User's Guide, The MathWorks, Natick, MA (1995).

22. Goldberg, D. E., Genetic Algorithms in Search, Optimization, and Machine Learning, Addison-Wesley, Reading, MA (1989).

23. Hsiao, F. H., Chen, C. W., Liang, Y. W., Xu, S. D., and Chiang, W. L., "T-S fuzzy controllers for nonlinear interconnected systems with multiple time delays," IEEE Transactions on Circuits \& Systems-I, Vol. 52, No. 9, pp. 1883-1893 (2005).

24. Hsiao, F. H., Chen, C. W., Wu, Y. H., and Chiang, W. L., "Fuzzy controllers for nonlinear interconnected TMD systems with external force," Journal of The Chinese Institute of Engineers, Vol. 28, No. 1, pp. 175181 (2005)

25. Hsiao, F. H., Chiang, W. L., Chen, C. W., Xu, S. D., and Wu, S. L., “Application and robustness design of fuzzy controller for resonant and chaotic systems with external disturbance," International Journal of Uncertainty, Fuzziness and Knowledge-Based System, Vol. 13, No. 3, pp. 281-295 (2005).

26. Hsiao, F. H., Hwang, J. D., Chen, C. W., and Tsai, Z. R., "Robust stabilization of nonlinear multiple time-delay large-scale systems via decentralized fuzzy control," IEEE Transactions on Fuzzy Systems, Vol. 13, No. 1 , pp. 152-163 (2005).
27. Hsieh, T. Y., Wang, Morris H. L., Chen C. W., Chen, C. Y., Yu, S. E., Yang, H. C., and Chen, T. H., "A new viewpoint of s-curve regression model and its application to construction management," International Journal on Artificial Intelligence Tools, Vol. 15, No. 2, pp. 131-142 (2006).

28. Limanond, S. and Si, J., "Neural-network-based control design: An LMI approach," IEEE Transactions on Neural Networks, Vol. 9, pp. 14221429 (1998).

29. Lin, M. L. and Chen, C. W., "Application of fuzzy models for the monitoring of ecologically sensitive ecosystems in a dynamic semi-arid landscape from satellite imagery," Engineering Computations, Vol. 27, (2010). (In press)

30. Lin, M. L., Chen, C. W., Wang, Q. B., Cao, Y., Shih, J. Y., Lee, Y. T. Chen, C. Y., and Wang, S., "Fuzzy model-based assessment and monitoring of desertification using MODIS satellite imagery," Engineering Computations, Vol. 26, No. 7, pp. 745-760 (2009).

31. Lu, L. T., Chiang, W. L., Tang, J. P., Liu, M. Y., and Chen, C. W., "Active control for a benchmark building under wind excitations," Journal of Wind Engineering and Industrial Aerodynamics, Vol. 91, No. 4, pp. 469493 (2003).

32. Ma, X. J., Sun, Z. Q., and He, Y. Y., “Analysis and design of fuzzy controller and fuzzy observer," IEEE Transactions on Fuzzy Systems, Vol. 6, No. 1, pp. 41-51 (1998).

33. Slotine, J. J. E. and Li, W., Applied Nonlinear Control, Prentice Hall, Englewood Cliffs, NJ (1991).

34. Takagi, T. and Sugeno, M., "Fuzzy identification of systems and its applications to modeling and control," IEEE Transactions on Systems, Man \& Cybernetics, Vol. 15, No. 1, pp. 116-132 (1985).

35. Tanaka, K., Hori, T., and Wang, H. O., "A multiple Lyapunov function approach to stabilization of fuzzy control systems," IEEE Transactions on Fuzzy Systems, Vol. 11, pp. 582-589 (2003).

36. Tanaka, K. and Wang, H. O., Fuzzy Control Systems Design and Analysis: A Linear Matrix Inequality Approach, John Wiley \& Sons, NJ (2001).

37. Tsai, C. H., Chen, C. W., Chiang, W. L., and Lin, M. L., "Application of geographic information system to the allocation of disaster shelters via fuzzy models," Engineering Computations, Vol. 25, No. 1, pp. 86-100 (2008).

38. Utkin, V. I., Sliding Modes and their Application in Variable Structure Systems, MIR Publishers, Moscow (1978).

39. Wang, L. X., Adaptive Fuzzy Systems and Control: Design and Stability Analysis, Prentice Hall, Englewood Cliffs, NJ (1994).

40. Wang, W. J. and Lin, H. R., "Fuzzy control design for the trajectory tracking on uncertain nonlinear systems," IEEE Transactions on Fuzzy Systems, Vol. 7, No. 1, pp. 53-62 (1999).

41. $\mathrm{Xu}, \mathrm{S}$. Y. and Lam, J., "Robust $\mathrm{H}^{\infty}$ control for uncertain discrete-timedelay fuzzy systems via output feedback controllers," IEEE Transactions on Fuzzy Systems, Vol. 13, No. 1, pp. 82-93 (2005).

42. Yeh, K., Chen, C. Y., and Chen, C. W., "Robustness design of time-delay fuzzy systems using fuzzy Lyapunov method," Applied Mathematics and Computation, Vol. 205, No. 2, pp. 568-577 (2008).

43. Yoo, B. and Ham, W., "Adaptive fuzzy sliding mode control of nonlinear system," IEEE Transactions on Fuzzy Systems, Vol. 6, No. 2, pp. 315-321 (1998). 